\title{
Efecto de una intervención motriz basada en el método de descubrimiento guiado sobre los patrones básicos de movimiento de un niño de 9 años: estudio de caso
}

Morera-Castro, María; Arguedas-Víquez, Rita; Brabenec-Aguilar, Stephanie

Efecto de una intervención motriz basada en el método de descubrimiento guiado sobre los patrones básicos de movimiento de un niño de 9 años: estudio de caso

MHSalud, vol. 17, núm. 1, 2020

Universidad Nacional, Costa Rica

Disponible en: http://www.redalyc.org/articulo.oa?id=237061117003

DOl: https://doi.org/10.15359/mhs.17-1.3

Esta obra está bajo una Licencia Creative Commons Atribución-NoComercial-SinDerivar 3.0 Internacional. 
MHSALUD, ISSN: 1659-097X, 17(1), ENERO-JUNIO, 2020, PP 1-12

\title{
Efecto de una intervención motriz basada en el método de descubrimiento guiado sobre los patrones básicos de movimiento de un niño de 9 años: estudio de caso
}

\author{
Effect of A Motor Intervention Based on The Guided Discovery Method on The Basic Movement Patterns of A 9- \\ Year- Child: Case Study
}

Efeito de uma intervenção motora baseada no método da descoberta guiada sobre os padrões básicos de movimento de uma criança de 9 anos: estudo de caso

Maria Morera-Castro

Universidad Nacional, Escuela de Ciencias del Movimiento

DOI: https://doi.org/10.15359/mhs.17-1.3

Humano y Calidad de Vida, Costa Rica

Redalyc: http://www.redalyc.org/articulo.oa?

mmore@una.ac.cr id $=237061117003$

(iD http://orcid.org/0000-0003-2218-179X

Rita Arguedas-Víquez

Universidad Nacional, Centro de Investigación y Docencia

en Educación, Costa Rica

rita.arguedas.viquez@una.ac.cr

(iD http://orcid.org/0000-0002-4052-0874

Stephanie Brabenec-Aguilar

Universidad Nacional, Escuela de Ciencias del Movimiento

Humano y Calidad de Vida, Costa Rica

cafecitaste@hotmail.com

(iD http://orcid.org/0000-0002-0064-854X

Recepción: 28 Junio 2019

Aprobación: 02 Octubre 2019

\section{Resumen:}

El propósito de esta investigación fue determinar el efecto de una intervención motriz basada en el método de descubrimiento guiado sobre los patrones básicos de movimiento de un niño típico. Se contó con la participación de un infante de 9 años que se percibe físicamente activo, practica deportes con regularidad, asiste a la escuela y posee un estado de salud y cognitivo normal. La intervención se aplicó durante 6 semanas, 2 sesiones de evaluación pretest y postest, por medio del instrumento para la evaluación de los patrones básicos de movimiento, y 4 sesiones prácticas de 60 minutos, un día a la semana, durante 4 semanas. Para el análisis de resultados, se utilizó el porcentaje de cambio. Los resultados, luego de la intervención, mostraron mejorías en el porcentaje de cambio, en 4 de los de los patrones locomotores entre el $33.33 \%$ y $300 \%$; en los patrones manipulativos, entre el $37.5 \%$ y $900 \%$; así como un mejoramiento del $50 \%$, en el total de las destrezas locomotoras, y de 85,18\%, la totalidad de las destrezas manipulativas. A su vez, tuvo una mejoría global en PBM del $65.08 \%$. Se concluye que, luego de este estudio de caso, un modelo de intervención motriz basado en el método empleado puede ser una herramienta útil para mejorar las destrezas motoras básicas en la población infantil.

Palabras clave: desarrollo motor, método de aprendizaje, niñez, salud, pedagogía.

\section{Abstract:}

The purpose of this research was to determine the effect of a motor intervention based on the guided discovery method on the basic movement patterns of a typical child. The study involved a 9-year-old child who is physically active, plays sports regularly, attends school, and has normal cognitive and health status. The intervention was applied during six weeks, two pre-test and posttest evaluation sessions through the Instrument for the Evaluation of Basic Movement Patterns, and four practical sessions of 60 minutes, one day a week, for four weeks. For the analysis of results, the percentage change was used. The results after the intervention showed improvements in the percentage of change in four of the locomotive patterns between $33.33 \%$ and $300 \%$; in the manipulative patterns between $37.5 \%$ and $900 \%$; as well as an improvement of $50 \%$ in the total of locomotive skills and 
85.18\% in the total of manipulative skills. In turn, it had an overall improvement in PBM of 65.08\%. It is concluded that after this case study, a model of motor intervention based on the method used can be a useful tool to improve basic motor skills in children. KEYWORDS: motor development, learning method, childhood, health education, pedagogy.

\section{Resumo:}

O objetivo desta pesquisa foi determinar o efeito de uma intervenção motora baseada no método de descoberta guiada sobre os padrões básicos de movimento de uma criança típica. O estudo envolveu uma criança de 9 anos de idade que é fisicamente ativa, pratica esportes regularmente, frequenta a escola e tem estado cognitivo e de saúde normal. A intervenção foi aplicada durante seis semanas, duas sessões de avaliação pré-teste e pós-teste através do Instrumento de Avaliação de Padrões Básicos de Movimento e quatro sessões práticas de 60 minutos, um dia por semana, durante quatro semanas. Para a análise dos resultados, foi utilizada a mudança percentual. Os resultados após a intervenção mostraram melhora no percentual de mudança em quatro dos padrões locomotivos entre 33,33\% e 300\%; nos padróes manipulativos entre 37,5\% e $900 \%$; bem como melhora de $50 \%$ no total de habilidades locomotivas e $85,18 \%$ no total de habilidades manipulativas. Por sua vez, teve uma melhora global na PBM de 65,08\%. Conclui-se que, após este estudo de caso, um modelo de intervenção motora baseado no método utilizado pode ser uma ferramenta útil para melhorar as habilidades motoras básicas em crianças.

PalaVRaS-CHAVE: desenvolvimento motor, método de aprendizagem, infância, saúde, pedagogia.

\section{INTRODUCCIÓN}

El desarrollo psicomotriz a edades tempranas es fundamental para un equilibrado desenvolvimiento cognoscitivo, afectivo y conductual, así como favorece un progreso íntegro en la persona. Por ello, es importante que los patrones motores básicos $(\mathrm{PMB})$ se aprendan de una manera eficiente y eficaz, como fundamentos básicos para llevar a cabo destrezas más complejas (Barnett et al., 2016; O’Brien, Belton y Issartel, 2015) y desarrollar estilos de vida físicamente activos en años posteriores de la existencia (Lloyd, Saunders, Bremmer y Tremblay, 2014).

Es necesario recalcar que los PMB no son dependientes de la edad y no maduran de forma natural, es decir, se requiere un aprendizaje a través de instrucciones, demostraciones y práctica habitual (Valentini y Rudisill, 2004). Según Gallahue y Ozmun (2006), los PMB se pueden establecer en tres etapas: inicial, elemental y madura o eficiente; esta última, normalmente, se desarrolla alrededor de los 6 a los 8 años. En ese momento, la ejecución de los movimientos es automáticamente eficiente, controlada y coordinada, permitiendo un perfeccionamiento de estos.

Diversos autores concuerdan con que un desempeño maduro de los patrones básicos traerá consigo, en la persona, una mayor motivación, seguridad en sí misma por participar en actividades, mejor salud física al estar físicamente activa de forma habitual y el mejoramiento del desarrollo cognitivo, afectivo, social y motor. Por tanto, cuando alguien sabe cómo moverse en el nivel más básico, le es más sencillo realizar distintas actividades físicas (Okely, Booth y Patterson, 2001; Robinson y Goodway, 2009; Stodden, Langendorfer y Roberton, 2009). Un estudio metaanalítico sobre intervenciones en PBM en niñez concluye que programas, ya sea en la escuela o en la comunidad, en los cuales se incluyan experiencias de aprendizaje apropiados para el desarrollo de estos patrones, guiados por especialistas en educación física o maestros de aula altamente capacitados, mejoran significativamente la competencia de los patrones en esta población (Morgan et al., 2013).

Uno de los modelos más empleados en la enseñanza de la técnica deportiva, las destrezas y habilidades motoras es el comando o instrucción directa (Fanarioti, 2014); sin embargo, investigaciones evidencian que un uso más variado de los estilos de enseñanza puede propiciar una mayor participación, satisfacción, atención, autonomía, conductas apropiadas y respuestas motrices en las clases de educación física (Carreras-Duaigües y Giménez-Fuentes-Guerra, 2010; Cuellar-Moreno, 2016; Perlmann, 2015; Özgül, Atan y Kangalgil, 2019). Esa afirmación concuerda con otra indagación, en la cual se reportan diferencias estadísticamente significativas entre adolescentes del grupo experimental y el grupo control, con puntuaciones más altas de los primeros en satisfacción de la lección, motivación intrínseca, actividades 
metacognitivas y regulación identificada en las clases de educación física (Chatzipanteli, Digelidis y Papaioannou, 2015).

Una revisión sistemática sobre el uso de métodos de enseñanza de reproducción y producción por parte de profesionales en educación física indica que, a través de 15 países, predomina la implementación de los de reproducción y solo algunas veces se aplican el descubrimiento guiado y los estilos de descubrimiento convergentes (Chatoupis, 2018).

El descubrimiento guiado como estilo de aprendizaje permite la elaboración de sesiones, en función de las necesidades de alguien y no del profesorado ni de la persona mentora o guía, ofreciendo la posibilidad de, mediante una serie de preguntas o problemas, que quien ejecuta tome decisiones que le permitan descubrir la solución y así construir su propio aprendizaje (Mosston y Ashworth, 1986). Igualmente, fomenta la capacidad de innovación, creación, descubrimiento, pensamiento independiente, exploración y resolución de problemas de manera activa (Shieh y Yu, 2016).

Investigaciones efectuadas evidencian que el método de descubrimiento guiado favorece el aprendizaje del estudiantado en diferentes áreas educativas como las ciencias básicas y tecnología (Agu y Samuel, 2018), la química (Akani, 2017) y la resolución de problemas matemáticos (Herdiana et al., 2017; Simamora, Saragih y Hasratuddin, 2018). Asimismo, tal método incentiva una mayor participación del estudiantado, una mejor base para el aprendizaje y una mayor retención que otros métodos (Mardanparvar, Sabohi, Yousefi y Rezaei Dehghani, 2016).

El desarrollo de métodos como el descubrimiento dirigido, por parte que las personas profesionales en la educación, potencializa en el estudiantado el análisis lógico de preguntas, la valoración o utilidad de la información y la síntesis de conocimiento para la generación de nuevas soluciones, que promueven destrezas cognitivas como el pensamiento crítico, la creatividad y la resolución de problemas, vitales para el afrontamiento del diario vivir (Shane y Brendan, 2017). Sin embargo, a pesar de que el método por descubrimiento dirigido ha sido empleado a través de los años en los diferentes campos de la educación, como se comprueba en investigaciones citadas, en el área de la educación física y la motricidad, específicamente, se cuenta con evidencias mayores durante los años 80 y 90 . En ellas se reflexiona y propone la utilización de este método en la práctica docente o como reforma de los estilos tradicionales de enseñanza de la educación física (Contreras-Jordán, 1998; Delgado-Noguera, 1991; Mosston y Ashworth, 1993; Sánchez-López-Bañuelos, 1989). No obstante, se han encontrado pocas investigaciones científicas experimentales en las cuales se utilice el método como intervención en el área de la motricidad. Además, como se indica en revisiones sistemáticas, se requiere mayor cantidad de indagaciones en esta área, enfocadas en metodologías de intervención eficientes, sólidas y con pautas detalladas para el mejoramiento del desarrollo motor en la niñez, así como la participación de profesionales que las implementen (Morgan et al., 2013; Riethmuller, Jones y Okely, 2009). Por tanto, el propósito de este trabajo fue establecer el efecto de una intervención motriz basada en el método de descubrimiento guiado sobre los patrones básicos de movimiento de un niño físicamente activo.

\section{Metodología}

\section{Caso de estudio}

Este estudio de caso se sustenta como una premisa inicial de un modelo específico de intervención motriz, que permite, a través de un análisis individual basado en el método de razonamiento inductivo, generar conclusiones preliminares que comprueben su efectividad, para que, posteriormente, sea aplicado en investigaciones experimentales.

Este caso fue seleccionado debido a que presenta experiencias individuales de un niño ideal de 9 años de edad, que cursa el tercer grado escolar en la escuela pública en Costa Rica. Su estatura actual es de 130 
cm y su peso es de $30 \mathrm{~kg}$. Vive con su madre de 43 años, quien no realiza actividad física de forma regular. Se considera físicamente activo, realiza más de 60 minutos diarios de actividad física moderada-vigorosa y practica habitualmente el baloncesto, en un equipo deportivo de su cantón, y fútbol, en la liga menor de un equipo de primera división. Usualmente, lleva a cabo competiciones deportivas los sábados y domingos de cada semana. Ha sido una persona que practica deportes activamente desde los 4 años y en el nivel competitivo desde los 7 . El niño presenta una óptima condición de salud física y cognitiva. Igualmente, no tiene ninguna patología ni condición médica o lesión muscular que le impida participar en la intervención. Asimismo, en el ámbito educativo, asiste regularmente a su institución y presenta facilidad en el aprendizaje del inglés, habilidades matemáticas y destrezas de lectura. En relación con sus vínculos sociales, presenta una gran desenvoltura para socializar y compartir con sus pares, especialmente con varones. La mayor parte de su tiempo libre la dedica al deporte y los videojuegos. Previo a la intervención, el niño reporta no haber practicado los patrones motores básicos, durante sus clases de educación física.

Según la montaña del desarrollo motor (Clark y Metcalfe, 2002), este niño se encuentra en el lapso de adquisición de destrezas motoras de contextos específicos del baloncesto y fútbol, lo que supone un dominio eficaz o maduro de periodos inferiores del desarrollo, como lo son los patrones básicos de movimiento.

\section{Instrumento}

Para evaluar los PBM, se empleó el instrumento para la evaluación de los patrones básicos de movimiento (IPBM) (Jiménez, Salazar y Morera, 2013). Este permite evaluar el desempeño motor de 10 patrones básicos de movimiento, divididos en destrezas locomotoras (correr, saltar a distancia, brincar, galopar, deslizarse lateralmente) y manipulativas (apañar, lanzar por encima del hombro, batear, patear y rebotar); a través de un grupo de 6 características observables, que describen el gesto del movimiento en un desempeño maduro/ eficiente. Dicho desenvolvimiento se puntúa de 1 , si efectúa el criterio, y de 0 , si no lo realiza. Para cada patrón el puntaje oscila entre 0 y 12 puntos, en 2 intentos; las subescalas locomoción y manipulación son el promedio de los 5 patrones correspondientes y del valor total de la prueba del promedio de los 10 patrones. Además, tal prueba posee un criterio de referencia (9), para clasificar el desempeño de la persona como un patrón maduro/eficiente (mayor o igual a 9) o inmaduro (menor a 9).

En la validación del IPBM, por juicio de expertos de un $99 \%$ en la pertinencia de las características para evaluar cada patrón de movimiento básico, en una etapa madura de ejecución, una confiabilidad y objetividad aceptables del total de la prueba son de $\mathrm{R}=.92$ y $\mathrm{R}=.86$, respectivamente (Jiménez et al., 2013). Por ello, se considera que el IPBM es un instrumento válido y confiable para esta población en estudio.

\section{Procedimiento}

En este estudio de caso, los procedimientos se efectuaron conforme a la declaración de Helsinki. Inicialmente, se conversó con la madre del niño y se le entregaron el consentimiento informado y asentimiento del menor. Una vez obtenidas ambas firmas, se les aplicó una entrevista sobre actividades cotidianas de la vida del niño y los PBM. Es importante señalar que la intervención motriz se llevó a cabo fuera del contexto educativo del niño.

En una primera sesión, se efectuó el pretest del IPBM, en una zona recreativa comunal que cumpliera las dimensiones y según los protocolos establecidos por Jiménez, Salazar y Morera (2013):

- Se debe trabajar en una zona amplia, como en un planché de cemento o zona verde, que cuente con un espacio libre de al menos $30 \mathrm{~m}$ x $20 \mathrm{~m}$.

- La evaluación del desempeño de los patrones es de forma individual. 
- Se hace una demostración para cada uno de los patrones. Es importante rescatar que se muestra qué deben hacer, no cómo debe hacer cada patrón.

- La persona evaluada debe ejecutar cada patrón motor en 2 intentos, pueden ser seguidos o alternos.

- Para la medición de cada patrón, hay un requerimiento de materiales e instrucciones específicas.

- Se filma con videocámara a la persona ejecutando el patrón y, posteriormente, se evalúa su desempeño de la filmación. Esto con el fin de observar los criterios de evaluación detenidamente.

En las sesiones de la 2 a la 5, se ejecutó la intervención motriz. Cada sesión semanal tuvo una duración aproximada de 60 minutos y se dividió en los 3 momentos propuestos en la metodología de educación psicomotriz vivencial (Lapierre y Aucounturier, 1985). A continuación, se describe cómo se aplicaron estos 3 momentos en este estudio de caso:

a) Primer momento de actividad motriz espontánea: 10 minutos donde el niño seleccionó libremente la actividad que deseaba efectuar durante este tiempo, propiciando, así, experiencias únicas de creación, construcción y disfrute del movimiento, desde sus propias experiencias y autonomía.

b) Segundo momento de análisis perceptivo: 40 minutos de actividades lúdicas y tareas motrices, empleando el método de descubrimiento guiado. La persona investigadora formuló la sesión y la consecución de preguntas o problemas motrices para que el niño, por medio de la experimentación y el descubrimiento, ejecutara los PBM. Así mismo, se le brindaron pistas, palabras claves y feedback frecuentes, como elementos de formación con miras a la adquisición de los PBM.

Un ejemplo de cómo se trabajó con el niño:

Explicación general. En este espacio, he colocado balones de diferentes tamaños y formas, al igual que palos, raquetas de diferentes texturas y tamaños, bates y otros materiales que podás utilizar en cualquier momento.

Pregunta. Demostrame qué podés hacer con esos materiales. Respuesta. El niño realiza figuras de animales; construye torres con los materiales; ordena las bolas por tamaños, formas, colores; golpea las bolas con los diferentes materiales. ¡Muy bien!, contesta la persona investigadora.

Pregunta. ¿De qué formas podés sostener los palos, raquetas, bate? Respuesta. Con las manos, los pies, el hombro, la cabeza y el estómago. Pregunta. ¡Excelente! Y si necesitás golpear con fuerza, ¿con qué partes del cuerpo lo podés hacer? ¿Son las mismas o diferentes? Respuesta. Algunas son las mismas, como los pies y las manos, también con las rodillas.

Pregunta. Si tuvieras que golpear las bolas con los materiales, ¿¿de qué formas podés agarrar los materiales y golpearlas? Respuesta. Con una mano, con una mano encima de la otra, 2 manos paralelas, 2 manos juntas.

¡Lo estás haciendo muy bien! Pregunta. Si tuvieras que golpear las bolas con los materiales, ¿con cuál material se te hace más fácil golpear cada una? Respuesta. Luego de ejecutar varios intentos con los diferentes materiales, con el palo es más fácil una bola grande, con la raqueta grande la bola pequeña, el bate me fue difícil.

¡Genial! Pregunta. ¿Qué juego podrías crear en el cual tengás que batear?...

c) Tercer momento de intelectualización de lo vivenciado: 10 minutos de reflexión o realimentación para que el niño pueda, junto con la persona investigadora, identificar sus propias vivencias y aprendizajes. En este espacio, se retomó con el niño cómo se sintió en el nivel emocional, qué actividades le fue más fácil o difícil ejecutar, qué aprendió, se reformó la técnica de las destrezas vistas en esa sesión, se le aclararon consultas y se le pidió que indicara cuáles actividades le gustaron más y cuáles menos.

Cada sesión de la intervención enfatizó en los siguientes patrones:

- Sesión 1. Correr, saltar a distancia y brincar. 
- Sesión 2. Deslizarse lateralmente y galopar.

- Sesión 3. Lanzar por encima del hombro, atrapar y batear.

- Sesión 4. Rebotar y patear.

En la sexta sesión se efectuó el postest, utilizando el mismo instrumento de medición del pretest y el protocolo correspondiente.

\section{Análisis estadístico}

Por la naturaleza de la investigación, un estudio de caso, los resultados obtenidos fueron analizados en el nivel cualitativo y se empleó el porcentaje de cambio como medida comparativa entre los valores iniciales (pretest) y los finales (postest). Para cada patrón básico, subescala y total de la prueba, se empleó la siguiente fórmula $\%$ cambio $=[$ (promedio postest - promedio pretest $) /$ promedio pretest $]{ }^{*} 100$ para cada patrón. Se utilizó el programa Microsoft Office 365 Excel para hacer los cálculos de los porcientos.

\section{Resultados}

En la tabla 1, se presentan los resultados de los valores obtenidos en las destrezas locomotoras y manipulativas pre- y posttest, así como el total de las subescalas, total de la prueba y porcentaje de cambio. Igualmente, en esta misma tabla, se incluye el desempeño del niño de cada patrón, subescala y total, antes y después de la intervención. Cabe señalar que el valor máximo del IBM en puntuación por patrón es 12 y con valores superiores a 9 se considera que el patrón básico es eficiente y maduro (Jiménez et al., 2013). 
MHSALUD, ISSN: 1659-097X, 17(1), ENERO-JUNIO, 2020, PP 1-12 Morera-Castro, Arguedas-Víouez, Brabenec-Aguilar

TABLA 1

Porcentaje de cambio, desempeño y resultados por patrón, subescala y total, del niño en estudio

\begin{tabular}{lccccc}
\hline Patrón & Pretest & Desempeño & Postest & Desempeño $\%$ de cambio \\
\hline Correr & 7 & Inmaduro & 12 & Eficiente & 71,43 \\
Galopar & 9 & Eficiente & 12 & Eficiente & 33,33 \\
$\begin{array}{l}\text { Brincar } \\
\begin{array}{l}\text { Saltar a } \\
\text { distancia }\end{array}\end{array}$ & 2 & Inmaduro & 8 & Inmaduro & 300 \\
$\begin{array}{l}\text { Deslizamiento } \\
\text { lateral }\end{array}$ & 12 & Eficiente & 12 & Eficiente & 0 \\
\hline $\begin{array}{l}\text { Total de } \\
\text { subescala de } \\
\text { locomotoras }\end{array}$ & 7.2 & Inmaduro & 10.8 & Eficiente & 50 \\
\hline $\begin{array}{l}\text { Rebotar } \\
\text { Batear }\end{array}$ & 10 & Eficiente & 10 & Eficiente & 0 \\
$\begin{array}{l}\text { Apañar } \\
\text { Patear }\end{array}$ & 2 & Inmaduro & 10 & Eficiente & 400 \\
$\begin{array}{l}\text { Lanzar por } \\
\text { encima del } \\
\text { hombro }\end{array}$ & 7 & Inmaduro & 10 & Eficiente & 42,86 \\
\hline $\begin{array}{l}\text { Total de } \\
\text { subescala de } \\
\text { manipulativas }\end{array}$ & 5 & Inmaduro & 11 & Eficiente & 37,5 \\
\hline $\begin{array}{l}\text { Total de } \\
\text { prueba }\end{array}$ & 6,3 & Inmaduro & 10,4 & Eficiente & 65,08 \\
\hline
\end{tabular}

Como se puede observar en la Tabla 1, la valoración inicial del niño, los patrones de más bajo desempeño fueron brincar, batear y lanzar por encima del hombro y solo batear, seguidos por un desempeño medio en correr, saltar a distancia, apañar y patear, así como por un desenvolvimiento alto, maduro y eficiente únicamente en 3 patrones: galopar, deslizarse lateralmente y rebotar. Cabe señalar que para la edad en la que se encuentra el niño todos los PBM deberían estar en un estadio eficiente y maduro (Clark y Metcalfe, 2002).

Posterior a la intervención, los resultados denotan un rango de mejoría de entre el 33,33 \% y el $900 \%$, en 7 patrones: correr, brincar, saltar a distancia, batear, apañar, patear y lanzar por encima del hombro. Lanzar por encima del hombro, batear y brincar fueron los ejercicios más bajos (ver Tabla 1).

Asimismo, de los 10 patrones posteriores a la intervención, únicamente el de brincar se mantiene en un desempeño medio inmaduro; el resto de los PMB adquiere su maduración y se ejecuta eficientemente (ver Tabla 1).

Además, en el caso particular del patrón galopar, aunque en el pretest se denotó un desempeño eficiente del niño, luego de la intervención, logró el puntaje completo en ejecución, es decir, los 12 pts. Caso semejante fue el patrón de correr; en su valoración inicial, se encontraba como patrón inmaduro y, después, alcanzó la totalidad del puntaje (ver Tabla 1). 
Es importante aclarar, que los patrones de deslizarse lateralmente y rebotar no sufrieron cambios en el puntaje, posteriores a la intervención; sin embargo, estos fueron 2 de los 3 patrones en los cuales el niño ya presentaba una ejecución eficiente (ver Tabla 1).

En cuanto a los resultados por subescalas locomotora y manipulativa, el niño logra incrementar su desempeño de inmaduro a eficiente. De igual forma, en la valoración total inicial este infante se catalogaba como una persona con PBM inmaduros, situación que se modificó posterior a la intervención, convirtiéndose en alguien con PBM eficientes (ver Tabla 1).

\section{Discusión}

En esta investigación, se encontró que el niño en estudio, previo a la intervención, presentaba 7 patrones en el rango de inmadurez y únicamente 3 con un desempeño maduro (galopar, deslizarse lateralmente y rebotar), a pesar de que reporta ser físicamente activo y practicar 2 deportes. Estos resultados concuerdan con los encontrados por O’Brien et al. (2015), en los cuales adolescentes entre los 12 y los 13 años no mostraron el dominio de los PBM. Tales datos también se asemejan a los encontrados en investigaciones sobre el desempeño de PBM en la niñez y adolescencia (Hardy, Barnett, Espinel y Okely, 2013; Mukherjee, Jamie y Fong, 2017).

Luego de 4 semanas de intervención motriz de 60 minutos cada sesión, el niño logró mejorar el porcentaje de cambio en su desempeño de 4 patrones locomotores y 4 manipulativos de los 10 posibles. Cabe señalar que los 2 patrones faltantes, previos a la intervención, se encontraban catalogados como maduros. Estos resultados concuerdan con los encontrados en un estudio de una intervención comunitaria basada en PBM (Bardid et al., 2017). Sin embargo, difieren con los hallados por Jiménez-Díaz y Araya-Vargas (2010), en cuanto a que no encontrar cambios significativos en los patrones manipulativos, pero sí en los locomotores, tras un proceso de intervención en las clases de educación física.

En el caso particular de brincar, que a pesar de mejorar aún no alcanzó su madurez, los resultados concuerdan con los encontrados por Jiménez-Díaz y Araya-Vargas (2010), luego de la participación de 90 minutos de clases de educación física por 8 semanas. Este patrón, según Goodway, Crowe y Ward (2003), es uno de los que más tarda en surgir, por el grado de complejidad y dificultad, así como requiere mayor práctica y tiempo para su maduración.

Por su parte, los resultados de los porcentajes de cambio encontrados en esta investigación concuerdan con las conclusiones efectuadas en investigaciones previas (Bardid et al., 2017; Hardy et al., 2013; Mukherjee et al., 2017) y en el metaanálisis ejecutado por Morgan et al. (2013), en los cuales indican que experiencias de aprendizaje enfocadas en el desarrollo de PBM en la niñez mejoran, significativamente, la competencia de los patrones.

Es importante resaltar, como fortaleza de este estudio, que además de que la intervención fue mediada por una persona profesional en el área de la educación física, el principal foco de atención fue crear, implementar y comprobar la efectividad de un modelo de intervención motriz. Este, basado en el método de descubrimiento guiado, que tuviera pautas claras y momentos específicos propiciadores de la creación, construcción y mejoramiento de los PBM desde la autonomía del niño. Dichas recomendaciones metodológicas, abordadas en esta investigación (en las que se incluyan detalles de la intervención) y aplicadas por profesionales, fueron sugeridas en las revisiones sistemáticas dirigidas a la eficiencia de intervenciones para el mejoramiento del desarrollo motor en la niñez (Morgan et al., 2013; Riethmuller et al., 2009).

Por tanto, una posible explicación de los resultados de esta investigación y una fortaleza de ella fue el uso del método por descubrimiento guiado, el cual, por el proceso de motivación, conlleva actividades cognoscitivas, a través de los retos planteados, que requieren que la persona efectúe acciones físicas para obtener el logro, de manera que satisface el interés y las necesidades del ejecutante (Abarca, 2003). No es propicio dejar de lado el saber previo del niño, con el objetivo de que adquiera, mediante secuencias ordenadas progresivamente, un 
conocimiento más elaborado, lo que permite un desarrollo más complejo y evolucionado de ideas y patrones motores (Monereo et al., 2008).

\section{Conclusiones}

Se concluye de esta investigación que el logro de la característica principal del aprendizaje por descubrimiento, la promoción a que el niño aprenda por sí mismo, de manera autónoma y consciente, se cumple, debido al mejoramiento en el desempeño de los patrones locomotores y manipulativos que obtuvo el infante luego de finalizada la intervención. Para este estudio de caso, el aprendizaje por descubrimiento no solamente conllevó que el niño lograra adquirir una mejor base motriz, sino también que superara sus limitaciones normales previas a tal aprendizaje, que a su vez lo estimuló a pensar por sí mismo, incentivó su autoestima y seguridad, así como lo entusiasmó a buscar una resolución creativa a cualquier situación de reto en el proceso de aprendizaje.

Asimismo, se concluye, luego de esta investigación de 4 semanas, que los PBM no se llegan a dominar eficientemente de forma natural, requieren ser enseñados para su aprendizaje, maduración y minimización de dificultades motrices a largo plazo, lo cual permita una exitosa transición a destrezas más avanzadas o complejas.

Además, la metodología empleada para la intervención motriz admite reforzar en el profesorado la importancia de enseñar, a partir de la estimulación de las ideas previas, por medio de retos motrices que le lleven a transformar lo que ya sabía en un proceso de maduración del desempeño motriz, a una etapa más madura. También es preciso, a su vez, que el mediador desarrolle una metodología y materiales óptimos, que den paso a la exploración motivada por la curiosidad de la persona aprendiz y que esto estimule al estudiante para que llegue a descubrir por sí mismo cómo funcionan las cosas de manera activa y constructiva.

Este tipo de estudio abre la oportunidad en el nivel pedagógico, para tomar como referencia nuevas investigaciones, que involucren intervenciones motrices basadas en el descubrimiento guiado para el aprendizaje o mejoramiento de los patrones de movimiento. Estas darán paso a una mayor participación, vinculación y autonomía de la población infantil y la niñez en actividades que se propongan desde las familias, las escuelas o las comunidades.

Cabe resaltar que es necesaria mayor cantidad de investigaciones en esta área, para determinar los efectos en mayor escala, a partir de los resultados obtenidos. Esto incluiría una mayor muestra de sujetos, comparaciones entre cantidades de sesiones por semana y la duración total de la intervención, así como la retención y las transferencias de lo aprendido. Igualmente, se recomienda, para futuras indagaciones, evaluar, en la población de estudio, aspectos psicosociales como la afectividad y las relaciones, propiciados durante la intervención, pero que no fueron medidos directamente en este trabajo.

\section{REFERENCIAS}

Abarca Mora, S. (2003). Psicología de la motivación. EUNED.

Agu, P. A. \& Samuel, I. R. (2018). Effect of reversed jigsaw, TAI cooperative and guided discovery instructional strategies on basic science and technology students' interest and achievement. International Journal of Innovative Education Research, 6(2), 19-26. Recuperado de http://seahipaj.org/journals-ci/june-2018/IJIER/full/IJIER-J -4-2018.pdf

Akani, O. (2017). Effect of guiden discovery method of instruction and student's achievement in chemistry at the secondary school level in Nigeria. International Journal of Scientific Research and Education, 5(2), 6226-6234. Recuperado de http://ijsae.in/index.php/ijsae/article/view/94

Bardid, F., Lenoir, M., Huyben, F., De Martelaer, K., Seghers, J., Goodway, J. D. \& Denoninck, F. J. A. (2017). The effectiveness of a community-based fundamental motor skill intervention in children aged 3-8 years: Results of 
MHSALUD, ISSN: 1659-097X, 17(1), ENERO-JUNIO, 2020, PP 1-12

Morera-Castro, Arguedas-Víouez, Brabenec-Aguilar

the "Multimove for Kids" project. Journal of Science and Medicine in Sport, 20(2), 184-189. https://doi.org/1 0.1016/j.jsams.2016.07.005

Barnett, L. M., Stodden, D., Cohen, K. E., Smith, J. J., Lubans, D. R., Lenoir, M., Iivonen, S., Miller, A. D., Laukkanen, A., Dudley, D., Lander, N. J., Brown, H. \& Morgan, P. J. (2016). Fundamental movement skills: An important focus. Journal of Teaching in Physical Education, 35(3), 219-225. https://doi.org/10.1123/jtpe.2014-0209

Carreras-Duaigües, J. C. y Giménez Fuentes-Guerra, J. (2010). Metodología de enseñanza utilizada en la enseñanza del tenis durante la etapa de iniciación. Retos. Nuevas tendencias en Educación Física, Deportes y Recreación, 18, 60-65. Recuperado de https://recyt.fecyt.es/index.php/retos/article/view/34654/18765

Chatoupis, C. C. (2018). Physical education teachers' use of Mosston and Ashworth's teaching styles: a literature review. The Physical Educator, 75(5), https://doi.org/10.18666/TPE-2018-V75-I5-8292

Chatzipanteli, A., Digelidis, N. \& Papaioannou, A. G. (2015). Self-regulation, motivation and teaching styles in physical education classes: An intervention study. Journal of Teaching in Physical Education, 34(2), 333-334. h ttps://doi.org/10.1123/jtpe.2013-0024

Clark, J. E. y Metcalfe, J. S. (2002). The mountain of motor development: a metaphor. In J. E. Clark y J. H. Humphrey (eds.), Motor Development: Research and Reviews, 2, (pp.163-190). Reston, VA: National Association for Sport and Physical Education.

Contreras-Jordán, O. R. (1998). Didáctica de la educación física. Un enfoque constructivista. Barcelona, España: INDE Publicaciones.

Cuellar-Moreno, M. (2016). Effects of the command and mixed styles on student learning in primary education. Journal of Physical Education and Sport, 16(4), 1159-1168. https://doi.org/10.7752/jpes.2016.04186

Delgado-Noguera, M. A. (1991). Estilos de enseñanza en la Educación Física. Propuesta para una reforma de la enseñanza. Granada: ICE, Universidad de Granada.

Fanarioti, M. (2014). The influence of direct and indirect teaching method in the development of selected technical skills in the sport of football to children aged 12-14 years old. Journal of Physical Education and Sport, 14(3), 413-420. https://doi.org/10.7752/jpes.2014.03063

Gallahue, D. \& Ozmun, J. (2006). Understanding motor development: Infants, children, adolescents. New York: McGraw Hill.

Goodway, J., Crowe, H. \& Ward, P. (2003). Effects of motor skill instruction on fundamental motor skill development. Adapted Physical Activity Quarterly, 20(3), 298-314. https://doi.org/10.1123/apaq.20.3.298

Hardy, L. L., Barnett, L., Espinel, P. \& Okely, A. D. (2013). Thirteen-year trends in child and adolescent fundamental movement skills: 1997-2010. Medicine \& Science in Sports \& Exercise, 45(10), 1965-1970. https://doi.org/10. 1249/MSS.0b013e318295a9fc

Herdiana, Y., Wahyudin, I. \& Sispiyati, R. (2017). Effectiveness of discovery learning model on mathematical problem solving. AIP Conference Proceedings 1868, 050028, 2-8. https://doi.org/10.1063/1.4995155

Jiménez-Díaz, J. y Araya-Vargas, G. (2010). Más minutos de educación física en preescolares favorece el desarrollo motor. Pensar en Movimiento: Revista de Ciencias del Ejercicio y la Salud, 8(1), 1-8. https://doi.org/10.15517 /pensarmov.v8i1.442

Jiménez, J., Salazar, W. y Morera, M. (2013). Diseño y validación de un instrumento para la evaluación de patrones básicos de movimiento. Motricidad. European Journal of Human Movement, 31, 87-97. Recuperado de http:// www.redalyc.org/articulo.oa?id=274229586006

Lapierre, A. y Aucounturier, B. (1985). La simbología del movimiento: psicomotricidad y educación. Barcelona, España: Editorial Científico Médica.

Lloyd, M., Saunders, T., Bremer, E. \& Tremblay, M. (2014). Lont-term importance of fundamental motor skills: A 20-year follow-up study. Adapted Physical Activity Quarterly, 31(1), 67-78. https://doi.org/10.1123/apaq.201 3-0048

Mardanparvar, H., Sabohi, F., Yousefi, H. \& Rezaei Dehghani, A. (2016). Comparison the effect of teaching by group guided discovery learning, questions $\&$ answers and lecturing methods on the level of learning and information 
MHSALUD, ISSN: 1659-097X, 17(1), ENERO-JUNIO, 2020, PP 1-12 Morera-Castro, Arguedas-Víouez, Brabenec-Aguilar

durability of students. Dimonthly of Education Strategies in Medical Sciences, 8(6), 35-41. Recuperado de https ://www.sid.ir/en/journal/ViewPaper.aspx?id=570624

Monereo, C., Badia, A., Baixeras, M., Boadas, E. Castelló, I. G., Miguel, E., Monte, M. y Sebastiani E. M. (2008). Ser estratégico y autónomo aprendiendo. Unidades didácticas de enseñanza estratégica para la ESO. Barcelona, España: GRAÓ.

Morgan, P.J., Barnett, L. M., Cliff, D. P., Okely, A. D., Scott, H. A., Cohen, K. E. \& Lubans, D. R. (2013). Fundamental movement skill interventions in youth: A systematic review and meta-analysis. Pediatrics, 132(5), e1361-e1383. https://doi.org/10.1542/peds.2013-1167

Mosston, M. y Ashworth, S. (1986). La enseñanza de la educación física. Barcelona, España: Editorial Hispano Europea, S. A.

Mosston, M. y Ashworth, S. (1993). La enseñanza de la educación física: la reforma de los estilos de enseñanza. Barcelona, España: Editorial Hispano Europea, S. A. Recuperado de https://juliita.files.wordpress.com/2009/10/metodo logia-los-estilos-de-ensenanza-de-m-moston.pdf

Munkerjee, S., Jamie, L. C. T. \& Fong, L. H. (2017). Fundamental motor skill proficiency of 6 to 9-year-old singaporean children. Perceptual and Motor Sills, 124(3), 584-600. https://doi.org/10.1177/00315125177030 05

O’Brien, W. O., Belton, S. \& Issartel, J. (2015). Fundamental movement skill proficiency amongst adolescent youth. Physical Education and Sport Pedagogy, 21(6), 557-571. https://doi.org/10.1080/17408989.2015.1017451

Okely, A., Booth, M. \& Patterson, J. (2001). Relationship of physical activity to fundamental movement skills among adolescents. Medicine \& Science in Sports \& Exercise, 33(11), 1899-1904. https://doi.org/10.1097/00005768200111000-00015

Özgül, F., Atan, T. \& Kangalgil, M. (2019). Comparison of the command and inclusion styles of physical education lessons to teach volleyball in middle school. The Physical Educator, 76(1). https://doi.org/10.18666/TPE-201 9-V76-I1-8481

Perlman, D. (2015). Assisting preservice teachers toward more motivationally supportive instructions. Journal of Teaching in Physical Education, 34(1), 119-130. http://dx.doi.org/10.1123/jtpe.2013-0208

Riethmuller, A. M., Jones, R. A. \& Okely, A. D. (2009). Efficacy of interventions to improve motor development in young children: A systematic review. Pediatrics, 124(4), e782-e792. https://doi.org/10.1542/peds.2009-0333

Robinson L. E. \& Goodway J. D. (2009). Instructional climates in preschool children who are at-risk. Part I: object control skill development. Research Quarterly for Exercise and Sport, 80(3), 533-542. https://doi.org/10.5641/ 027013609X13088500159480

Sánchez-López Bañuelos, F. (1989). Bases para una didáctica de la educación física y deporte. Madrid, España: Gymnos.

Shane, P. \& Brendan, S. S. (2017). Including critical thinking and problem solving in physical education. Journal of Physical Education, Recreation \& Dance, 88(9), 43-49. https://doi.org/10.1080/07303084.2017.1367741

Shieh, C. J. \& Yu, L.A. (2016). Study on information technology integrated guided discovery instruction toward students learning achievement and learning retention. Eurosia Journal of Mathematics, Science \& Technology Education, 12(4), 833-842. https://doi.org/10.12973/eurasia.2015.1554a

Simamora, R. E., Saragih, S. \& Hasratuddin (2018). Improving students' mathematical problem solving ability and self-efficacy through guided Discovery Learning in local cultural context. International Electronic Journal of Mathematics Education, 14(1), 61-72. https://doi.org/10.12973/iejme/3966

Stodden, D., Langendorfer, S. \& Roberton, M. (2009). The association between motor skill competence and physical fitness in youth adults. Research Quarterly for Exercise and Sport, 80(2), 223-229. https://doi.org/10.5641/027 013609X13087704028318

Valentini, N. \& Rudisill, M. E. (2004). Motivational climate, motor-skill development, and perceived competence: Two studies of developmentally delayed kindergarten children. Journal of Teaching in Physical Education, 23(3), 216-234. https://doi.org/10.1123/jtpe.23.3.216

\section{BY-NC-ND}

